\title{
The Use of IVIG in Neurological Disease
}

\author{
Arnold l. Levinson \\ University of Pennsylvania School of Medicine, Philadelphia, PA
}

\section{INTRODUCTION}

Garnma-globulin therapy has been practiced since the turn of the century, when clinicians used heterologous protein preparations to provide passive immunity to exposed unimmunized hosts. The need for a $\gamma$-globulin preparation capable of transferring broad-based humoral immunity was recognized in the early 1950s following the description of agammaglobulinemia by Bruton. Since that time, tremendous progress has been made in the manufacturing of safe and efficacious $\gamma$-globulin products, culminating in the development of agents that can be administered intravenously. Such preparations contain a large array of antibody specificities, having been prepared from the blood of thousands of healthy donors.

The remarkable irony of the intravenous immunoglobulin (IVIG) story is that an agent developed to satisfy the needs of the immunedeficient patient appears to have therapeutic effects in patients with autoimmune diseases. The efficacy of IVIG has been established in several autoimmune disorders, including childhood and adult immune thrombocytopenia (1), other immune cytopenias (2), acquired factor-VIII deficiency (3), and Kawasaki disease (4). The efficacy of IVIG is also currently being explored in patients with rheumatoid arthritis, systemic lupus erythematosus, systemic vasculitis, and bullous pemphigoid and a number of neurological diseases in which

IVG Therapy Today - Ed: M. Ballow 1992 The Humana Press Inc. - Totowa, NJ 
autoimmunity or aberrant immune responses have been established. The use of IVIG in such neurological diseases is the subject of this communication.

\section{Mrasthenia Ganavis}

Myasthenia gravis (MG) is a disorder characterized by weakness of striated muscles. The hallmark of this muscle weakness is its tendency to fluctuate over time, particularly with repetitive usage. $\mathrm{MG}$ is now viewed as a prototypic autoimmune disease, one in which autoimune effector mechanisms and target have been elucidated (reviewed in ref. 5). The disease is caused by IgG antibodies that react with the $\alpha$ chain of the nicotinic acetylcholine receptor $(\mathrm{AChR})$. Circumstantial evidence incriminating these antibodies in the pathogenesis of $\mathrm{MG}$ includes the finding of anti-AChR antibodies in the serum of $80-90 \%$ of MG patients, development of neonatal MG in some infants born to myasthenic mothers, and the deposition of IgG and complement at the postsynaptic myoneural junction. The transfer of disease from patient to mouse by injection of patient serum IgG provided formal proof of the culpability of anti-AChR antibodies.

Anti-AChR antibodies impair neuromuscular transmisssion by several potential mechanisms (reviewed in ref. 5). They damage the postsynaptic membrane by a complement-dependent mechanism. They markedly diminish AChR density at the postsynaptic membrane by accelerating the degradation of receptor protein. Some of the anti-AChR antibodies block cholinergic binding sites on the AChR $\alpha$ chain, but most are directed at a more $\mathrm{N}$-terminal epitope. $\mathrm{Fi}$ nally, it is possible that a minor subset of these autoantibodies decrease AChR synthesis.

Anticholinesterase agents represent the mainstay of treatment for MG patients. They increase the amount of acetylcholine available to the reduced number of receptors. This enhances the likelihood of sufficient ligand-receptor interactions to effect neuromuscular transmission. A modality that has proven to be useful, particularly as a stopgap measure, is plasmapharesis. Presumably, antibodies and other kinds of proinflammatory factors, including complement components, are removed. Corticosteroids and immunosuppressive agents are reserved for patients 
who have resistant disease. Thymectomy seems to be particularly beneficial to patients with disease of relatively short duration, young females, and individuals who are found to have abundant germinal centers in their thymic glands.

As is true for most trials of IVIG in neurological disorders, its use in MG is best described as preliminary and unproven. In the first reported study (6), five patients with severe, generalized $\mathrm{MG}$ were studied. Two patients were given $200 \mathrm{mg} / \mathrm{kg}$ and three were given $400 \mathrm{mg} / \mathrm{kg}$ IVIG on each of five consecutive days. All the patients showed improved neurological scores by days 10-15 (loosely meaning that they had increased strength by this time) and an increased vital capacity by day 25 . In three cases discontinuation of nasogastric feeding and assisted ventilation was possible. There was a decrease in anti-AChR antibody titers on days 10 and 15 , with a return to pretreatment levels by day 25 . These initial observations have been confirmed in an expanded study (7).

In a second study (8), originally reported in preliminary form (9), patients were treated with either an intact (7-s) IgG fraction of IVIG or a pepsin-treated 5-s fraction. The first four patients were treated with six infusions of $20 \mathrm{~g}$ of IVIG over a 2 -wk period. This was followed by a 4-wk washout period and crossover to the other preparation. Patients who initially received the 7-s preparation subsequently received the 5 -s preparation. Treatment with the 7-s preparation was associated with what was viewed as significant clinical improvement and a slight decrease in the anti-AChR antibody titers, whereas no improvement was observed after treatment with the 5 -s preparation.

In a more comprehensive study (10), IVIG was administered to 12 patients ranging in age from $15-70 \mathrm{yr}$ who had been ill for varied periods of time. Some of these patients had already undergone thymectomy, some were being treated concurrently with prednisone, and a few were being treated with azathioprine. There was a statistically significant improvement in clinical status following administration of $400 \mathrm{mg} / \mathrm{kg}$ IVIG on five consecutive days. The maximum improvement in these patients was observed in a mean of $8.6 \mathrm{~d}$ and improvement was maintained for a mean duration of $52 \mathrm{~d}$.

In a more recent trial (11), all of five patients treated with 400 $\mathrm{mg} / \mathrm{kg}$ IVIG improved; striking improvement was noted within 2$20 \mathrm{~d}$ in four of these patients. Concurrent study of hematological and immunological laboratory parameters showed a decrease in the 
white-blood-cell count by day 1 and an absolute decrease in neutrophils and a relative decrease in lymphocytes over a period of $6 \mathrm{~d}$ post-therapy. The investigators observed an increase in the percentage of peripheral blood surface immunoglobulin-bearing cells, made up mainly by surface IgG-bearing cells. This percentage was much higher than is typically seen in the blood of normal or myasthenic subjects. This suggested that the IgG infused might be binding to a population of cells that have receptors for the $F c$ fragment of $I g G$, leading to a spuriously elevated value of surface IgG-positive lymphocytes. In addition, an increased percentage of CD16 $6^{+}$lymphocytes was also observed, as well as a slight but statistically significant decrease in the CD4/CD8 ratio.

The results of these trials of IVIG in MG suggested that IVIG therapy was associated with rapid clinical improvement in patients with moderate to severe disease, independent of whether these patients had undergone thymectomy or had been treated concurrently with corticosteroids or azathioprine. Improvement was seen in many of the patients and occurred early in the treatment course. In some patients, improvement was sustained over a period of several weeks. The mechanism of action of this apparent salutary effect is unknown, but likely relates to one or more of the actions of IVIG described in other autoimmune disorders. Studies in patients with idiopathic thrombocytopenia pupura (ITP) (1) indicate that IVIG interferes with Fcy receptor mediated clearance of IgG-coated particles by the monocyte/macrophage lineage of cells. This effect could be important in $\mathrm{MG}$, since macrophages may contribute to the destruction of postsynaptic membranes if targeted by IgG anti-AChR antibodies. IVIG also interferes with the uptake of $\mathrm{C} 3$ on immunoglobulin-sensitized target cells (12). This action could inhibit complement-mediated destruction of the postsynaptic membrane.

IVIG administration might suppress autoantibody production via its nonspecific immunomodulatory activity. In vitro studies have underscored broad-based immunoregulatory actions of IVIG. IVIG inhibits (1) proliferation of peripheral blood mononuclear cells stimulated with PHA or allogeneic cells (13) and (2) the differentiation of $B$ cells to antibody-secreting cells following their stimulation with the polyclonal B-cell activator pokeweed mitogen (14). These effects are mediated by the Fc portion of the molecule, but their cellular basis is not yet settled. An interesting possibility, first suggested by studies in a patient with acquired factor-VIII deficiency (3), is that 
IVIG may downregulate autoantibody responses by providing a source of antiidiotypic antibodies. Autoantibody responses, such as the antibody responses to exogenous antigens, appear to be regulated by an idiotype/anti-idiotype network (15). According to the network theory, stimulation of the immune system leads to a conventional antibody response, which in turn elicits the production of antibodies directed against determinants in and around the antigen binding site of the the conventional antibodies. Indeed, a reverberating circuitry of antiantibodies, antiantiantibodies, and so on has been demonstrated. Some of the second-order antibody molecules, i.e., antiidiotypic molecules, have been shown to have specific suppressor activity. There is evidence to suggest that the failure to make an appropriate antiidiotypic response leads to the production of autoantibodies (16). In the past year, the list of antiidiotypic antibodies found in commercial IVIG has been expanded to include antibodies reactive with anti-DNA, antithyroglobulin and antiintrinsic antibodies $(17,18)$. Thus IVIG, which is prepared from the blood of thousands of healthy volunteers, might provide a source of antibodies directed against major crossreactive determinants on certain families of autoantibodies and provide a source of regulatory antiidiotypic antibodies lacking in patients with certain autoimmune diseases. However, the improvement observed in IVIGtreated MG patients has not been consistently associated with a change in anti-AChR antibody titers. This makes antiidiotypic antibodies an unlikely mechanism. Moreover, antibodies reactive with idiotypes on antiAChR antibodies have not been detected in IVIG preparations. Furthermore, there is conflicting data on whether anti$\mathrm{AChR}$ antibodies express a major crossreactive idiotype.

The successful treatment of children with Kawasaki disease (KD) (4) has underscored potential effects of IVIG on immune activation and consequent release/activity of cytokines. Leung and his coworkers have provided striking evidence for immunoregulatory imbalance in the acute form of this disease, highlighted by augmented T-cell, B-cell, and macrophage activation (reviewed in ref.19). T-cell and macrophage activation are signaled by the finding of increased levels of $\gamma$-interferon and tumor necrosis factor in the serum and increased spontaneous secretion of IL- 1 by circulating monocytes of patients with acute KD. Their results indicate that IVIG repairs the immunregulatory imbalance in $\mathrm{KD}$ by (1) increasing the numbers of $\mathrm{CD}^{+}$suppressor $\mathrm{T}$ cells and (2) decreasing the number of activated 
$T$ cells and the number of B-lineage cells spontaneously secreting antibodies. At present there is no evidence that proinflammatory cytokines are involved in the pathogenesis of $M G$. Thus this mechanism is not likely to be important in the mechanism of action of IVIG in MG.

\section{EPILEPSY}

Intractable childhood epilepsy is a disease that does not respond to the usual anticonvulsant therapies. The etiology of this disorder is not clear, but there is a suggestion that an aberrant immune reactivity may be involved in some forms of epilepsy. Eleptogenic foci or seizure activity have been elicited in experimental animals with antiserum to various brain constituents administered directly into the brain, raising the possibility that some patients may be suffering from an autoimmune process (20). Patients with infantile spasms have been reported to show increased intrathecal IgG production (21). This observation indicates that at least some patients may have an abnormal central-nervous system (CNS) humoral response, but falls short of linking this abnormality and the pathogenesis of disease.

Seizure disorders have been associated with immunodeficiency states, most notably selective IgA deficiency (22) and isolated IgGsubclass deficiency $(23,24)$. This has led to the hypothesis that some patients may be suffering from a chronic viral infection of the meninges, as is seen in a small subset of patients with congenital agammaglobulinemia. It must be pointed out, however, that there is no evidence to support this postulate. Nevertheless, treatment of a limited number of patients with intractable epilepsy has been attended by improvement.

Initial trials of $\gamma$-globulin in intractable epilepsy were carried out in France. In one study, seven of 10 patients showed improvement in EEG activity (25). In a second study, improvement in seizure activity as well as EEG was reported in seven of nine patients (26). Administration of TVIG in a dosage of $100-200 \mathrm{mg} / \mathrm{kg}$ was associated with improvement in four of 18 patients with uncharacterized seizure disorders (27). A follow-up crossover study (28) examined the efficacy of intact IgG vs $\mathrm{F}\left(\mathrm{ab}^{\prime}\right)_{2}$ administered intravenously to such patients. Improvement was seen predominantly with 
the native product, and two patients with the Lennox-Gastaut syndrome improved dramatically. Treatment consisted of $100 \mathrm{mg} / \mathrm{kg}$ every 3 wk for a period of $6 \mathrm{mo}$. In another study (29), children with infantile spasms, including some with the Lennox-Gastaut and West syndromes, were treated with a total of $2 \mathrm{~g}$ of IVIG administered in divided doses over 2 or $5 \mathrm{~d}$. Resolution of symptoms was seen in three of 12 patients; another two showed clinical improvement. Patients with postencephalitic seizures have also shown improvement in clinical status following treatment with TVIG (30). Four of eight patients treated with $100-200 \mathrm{mg} / \mathrm{d}$ every $2 \mathrm{~d}$ showed $>75 \%$ reduction in seizures. Interestingly, an overall reduction in intrathecal immunoglobulin synthesis was observed in these patients. The latter observation raises the possibility that IVIG exerted an immunomodulatory role on CNS humoral immune reactivity. Finally, two of five children with intractable seizures responded partially to a regimen of $1 \mathrm{~g}$ of IVIG on two consecutive days with repeat doses every 3-4 wk (31).

The possible contribution of underlying humoral immune deficiency to epilepsy was underscored in a study by Duse et al. (23). Patients were treated with $400 \mathrm{mg} / \mathrm{kg}$ IVIG every $21 \mathrm{~d}$. Interestingly, improvement was seen only in those children with an underlying IgG-subclass deficiency. Six of 12 patients were found to have $\mathrm{IgG}_{2}$ subclass deficiency. Three of these patients had an associated $\mathrm{IgG}_{4}$ subclass deficiency, and two had $\operatorname{IgG}_{4}$ deficiency alone. Thus two-thirds of the patients studied had an associated IgG-subclass deficiency. Improvement, based on EEG monitoring and assessment of clinical seizure activity, was seen in five patients, and all five had $\mathrm{IgG-}_{2}$ subclass deficiency. The initial findings were later confirmed in an expanded follow-up study (24).

The above uncontrolled studies suggest a role for IVIG in patients with intractable epilepsy, but the value of this therapeutic modality will require validation in controlled studies. There is some evidence that administration of $\gamma$-globulin intravenously affects the dynamics of immunoglobulin on the other side of the blood-brain barrier. Yet it remains to be determined if the administered $\gamma$-globulin actually traverses the blood-brain barrier. This would require that the blood-brain barrier be abnormally permeabilized in this group of patients. As yet there is no striking evidence for this abnormality, even though occult infection has been cited as a potential etiological cause of intractable epilepsy. 


\section{Demyelinating Polyneuropathies}

The disorders in this group are characterized pathologically by segmental demyelination and sparing of axons in peripheral nerves. The electrodiagnostic features of the demyelinating polyneuropathies are multifocal slowing and conduction block, which may be transient and associated with rapid remyelination. Based on their presumed immunopathogenesis, three types of demyelinating neuropathies have been considered as candidates for a trial of IVIG.

\section{Guilliar-Barre Syndrome (GBS)}

GBS is an acute progressive polyradiculopathy with symptoms of motor weakness of the extremities and bulbar and facial musculature. Patients have reduced-to-absent deep tendon reflexes and a variable sensory deficit. The cerebrospinal fluid is characterized by elevated protein with few or no inflammatory cells. Pathologically, lesions show segmental demyelination and mononuclear cell infiltrates. Severe demyelination is associated with the infiltration of activated macrophages in the endoneurium. The development of GBS after viral infection or vaccination has suggested an etiological role for viruses in this disease. The majority of patients demonstrate a conduction slowing or block, and recover. A small percentage of patients has a more fulminant course complicated by ventilatory failure and delayed recovery.

Several observations indicate that autoimmune reactivity is central to the pathogenesis of GBS (reviewed in ref. 32). An experimental model, called experimental autoimmune neuritis (EAN), which has the clinical, electrophysiological, and histological features of GBS, can be induced by immunizing animals with myelin or neuritogenic components of myelin, e.g., $\mathrm{P}_{0}, \mathrm{P}_{2}$, or galactocerebroside. The disease can be transferred from immunized animals with $\mathrm{CD} 4+\mathrm{T}$ cells that are reactive with myelin protein $\mathrm{P}_{2}$. Humoral mechanisms may also be important, since intraneural transfer of serum from EAN animals or GBS patients into animal recipients is associated with demyelination and/or conduction block. Deposits of immunoglobulin on peripheral nerve tissue have been widely reported in the literature, but their importance is minimized by the detection of such deposits in nondemyelinating poly. neuropathies and the fact that immunoglobulins may bind 
nonspecifically to peripheral nerves. On the other hand, more recent studies hint at the potential etiological role of complement-fixing antibodies in GBS. Moreover, the involvement of humoral factors in the pathogenesis of GBS is consistent with the beneficial effects of plasmapharesis in patients with this disease (33).

There has been one uncontrolled study of IVIG treatment in GBS (34). The patients had severe disease, having failed treatment with plasmapharesis. Six patients were treated every $3-4$ wk with $400 \mathrm{mg} / \mathrm{kg}$ IVIG. Improvement in muscle strength was observed, in some within a 48 -h period. Improvement was selflimited, necessitating repeat courses. There is currently an ongoing trial of IVIG in GBS in the Netherlands.

\section{Chronic Inflammatory Demyelinating Polyneuropathy (CIDP)}

This syndrome is thought to represent a variant of GBS. The pathological hallmark is scattered inflammatory lesions in nerve roots, plexi, trunks, and terminals. CIDP affects all age groups, but is seen most commonly in the fifth and sixth decades of life. There is rarely a history of antecedent (few weeks) infection, or receipt of biological materials. This diagnosis requires exclusion of patients with (1) a family history suggestive of polyneuropathy and (2) evidence of diabetes, renal insufficiency, connective-tissue disease, monoclonal gammopathy, porphyria, or exposure to heavy metals or hexacarbons. The disease follows a subacute, chronic progressive, or relapsing and remitting course. The overall prognosis is worse than that for GBS. The clinical picture is dominated by motor and sensory deficits. As is true for GBS, the spinal fluid is notable for an elevated protein, along with few or no cells. Nerve biopsy reveals segmental demyelination and, in $50 \%$ of the cases, perivascular mononuclear infiltrates.

The suspicion that CIDP is immune-mediated relates to (1) the finding of complement-fixing antimyelin antibodies in some patients, (2) the observation of impaired conduction velocity in experimental animals following transfer of IgG from CIDP patients, (3) the deposition of immunoglobulin on sural-nerve biopsies of CIDP patients, (4) the prominent class II MHC antigen staining of nerve fascicles, capillary endothelial cells, and perineurium, and (5) the elicitation 
of disorders with similar clinical and pathological features in experimental animals following immunization with peripheral nerve components or after intraneural injection of hyperimmune serum from immunized animals (reviewed in ref. 32). Therapeutic approaches include corticosteroids, azathioprine, cyclosporine, and plasmapharesis.

In an early study (35), patients with CIDP were treated with fresh-frozen plasma infused at a dose of $0.1 \mathrm{~L} / \mathrm{kg} /$ body wt over a period of 5-7 d. Clinical improvement was observed in 13 of 17 cases, with the first signs of improvement noted within $8 \mathrm{~d}$ of the initiation of therapy. The beneficial effect of therapy persisted for 4 mo after a single treatment course in four patients. In the remaining nine patients, the effects of treatment were short-lasting. Plasma infusions were repeated in all patients when improvement was not sustained or they showed further deterioration. Nine of these patients were subsequently treated with IVIG, $400 \mathrm{mg} / \mathrm{kg}$, on five consecutive days. A beneficial effect was observed in eight, with a similar temporal pattern of improvement. The single patient who did not benefit from IVIG likewise had not improved following plasma infusion. IVIG dosing intervals were then established empirically, and with treatment continued for 6 mo to $2 \mathrm{yr}$. Nerve conduction was improved posttherapy in several of the patients. The prompt response seen in these patients led the authors to postulate that weakness observed was a result of a functional axonal defect. The authors attributed the improvement to therapy, since none of the patients had shown any improvement in the 2 mo prior to treatment, 10 of the patients had never experienced spontaneous remission, and the temporal pattern of response deterioration following cessation of therapy was most consistent with a treatment effect. The beneficial effects of IVIG were confirmed in a later uncontrolled study (36) that employed a similar dosing regimen. Here, improvement in muscle strength and reflexes was sometimes seen as early as $48 \mathrm{~h}$ after commencement of therapy. IVIG had a similar salutary effect in an additional patient with CIDP hypogammaglobulinemia (37).

In the only randomized trial to date (38), IVIG was discontinued in seven disabled patients who had previously responded to this form of therapy. The patients were selected because they deteriorated clinically when IVIG was discontinued and were not on corticosteroids, other immunosuppressives, or plasmapharesis. The mean treatment duration had been 27 mo; mean treatment interval, 2 
wk; and mean dosage, $0.4 \mathrm{~g} / \mathrm{kg}$ body wt $2 \mathrm{wk}$ prior to cessation of therapy. The patients were randomized to either IVIG or albumin placebo once deterioration became apparent after discontinuation of therapy. They were then crossed over to the second trial treatment upon further deterioration. IVIG was administered at a dose of $400 \mathrm{mg} / \mathrm{kg} / \mathrm{d}$ for a total of $5 \mathrm{~d}$.

Clinical improvement, assessed by a quantiative disability-rating scale, was observed in all seven patients by day 8 after onset of treatment with IVIG. By contrast, no patient improved after the placebo trial and two actually deteriorated. Discontinuation of IVIG and placebo were associated with clinical deterioration in a mean of 6.4 and $1.3 \mathrm{wk}$, respectively. Clinical improvement was associated with a trend toward improvement in conduction velocity. Thus, in this limited controlled and double-blinded study, IVIG treatment was clearly associated with clinical improvement. Although this trial validated the efficacy of IVIG, it is not clear how well the results can be transferred to the entire group of patients with CIDP. Inclusion in the study was biased, since patient selection depended upon an earlier treatment response.

\section{Demyelinating Polyneuropathy Associated with Monoclonal Gammapathy}

These patients share clinical, histological, and electrophysiological features of CIDP, but in addition have a serum monoclonal protein, usually of an IgM $\kappa$ isotype, and no evidence of malignant disease. The monoclonal proteins often show specificity for peripheral-nerve constituents, including specific glycoproteins (myelinassociated glycoprotein, or MAG) and glycolipids (reviewed in refs. 32 and 39). It is widely believed that these autoantibodies are etiological in this syndrome. This is supported by (1) detection of the deposition of immunoglobulin of appropriate heavy-and light-chain isotypes in affected sural nerves, (2) demyelination of cat sciatic nerve following intraneural injection of monoclonal anti-MAG antibodies, and (3) the beneficial effect of plasmapharesis in some patients.

Cook (40) reported two patients who had been severely disabled by CIDP and IgM paraproteinemia over a period of 3 yr. One patient had failed treatment with steroids and therapy with cytotoxic agents, and plasmapharesis had been complicated by sepsis. The second patient failed to respond to trials of prednisone (after an ini- 
tial transient response), azathioprine, and plasmapharesis. One patient had a monoclonal IgM $\kappa$ protein with specificity for GM1 and GM1n gangliosides, whereas the second had a monoclonal IgM $\lambda$ paraprotein that reacted with MAG. Both patients were treated with IVIG, $2 \mathrm{~g} / \mathrm{kg}$, administered in five daily doses (patient 1) and three daily doses (patient 2). Both patients showed dramatic clinical improvement within 8-10 days. The improvement was shortlived and required repeat treatments. Each treatment with this dose of IVIG was associated with clinical recovery in strength and sensation. Substitution of one-fifth the dose in patient 1 was associated with some deterioration. Reinstitution of the higher dose was again associated with clinical improvement.

As yet the mechanism of action of IVIG in patients with GBS or either of the types of CIDP is unknown. Postulated effects relate largely to the immunomodulatory actions of this agent. In one study (41) treatment of GBS and CIDP patients with IVIG led to a fall in titer of antibodies reactive with a neuroblastoma cell line. The detected antibody was considered to be clinically relevant since several patient sera lost activity when adsorbed with human sciatic nerve tissue. IVIG neutralized the binding of IgM antibodies to the target neuroblastoma cell line in one instance. This raised the possibility that IVIG contains antibodies recognizing crossreactive idiotypes on putative pathological autoantibodies. It is also possible that IVIG might interfere with destruction of peripheral nerve myelin by IgG targeted macrophages or complement-binding immunoglobulins.

\section{Miscellaneous}

Multiple sclerosis (MS) is an inflammatory demyelinating disease that has been suspected of having an autoimmune or infectious etiology (reviewed in ref. 42). Based largely on an experimental model of MS (experimental allergic encephalomyelitis), T-cell sensitivity to myelin constituents has received most attention. Patients also produce antimyelin antibodies, although the significance of this finding is unclear. A number of immunomodulating therapies have been used in MS, including immunosuppressive agents, interferon, and the mainstay of therapy, ACTH or corticosteroids. A major problem in evaluating the response to any therapy in this disease re- 
lates to its unpredictable natural history and the difficulty in quantifying objective and subjective signs of clinical activity.

There have been only limited trials of IVIG in MS patients. In an early study (43), IVIG was administered to 20 patients at 2 -mo intervals for $1 \mathrm{yr}$. Significant clinical improvement was noted, with most of the benefit seen in symptoms relating to the cranial nerves and the extremities. In a second study (44), patients who had been treated with intramuscular $\gamma$-globulin for $3 \mathrm{yr}$ were switched to IVIG for $4 \mathrm{yr}$. Whereas 11 patients continued to deteriorate, nine were unchanged and 11 improved clinically. In a more recent study (45), patients with MS received IVIG at the start of disease or at the time of relapse. IVIG was administered in a total dose of $2.5-5 \mathrm{~g}$ in daily divided doses and repeated $14-21 \mathrm{~d}$ thereafter. Improvement in neurological deficits and subjective symptoms was observed within $24 \mathrm{~h}$ and was maintained, on average, for $14 \mathrm{~d}$. Four patients were maintained on IVIG every $3-4$ wk with apparent retardation of the progression of the disease and reduction in concomitant medications, including corticosteroids.

Polymyositis is an inflammatory disease of striated muscles characterized by proximal muscle weakness, mononuclear cell infiltrates, and characteristic electrodiagnostic changes on electromyogram (reviewed in ref. 46). Although a characteristic autoantibody (Jo-1) is seen in a subset of patients and disease in childhood is associated with vasculitis, the bulk of evidence highlights the potential importance of an autoaggressive T-cell-mediated immune destruction of muscle fibers. Roifman et al. (47) reported their experience on IVIG treatment of a 15-yr-old girl who had been debilitated with polymyositis for several years. Her disease had consistently flared upon tapering of prednisone, and she had failed a trial of pulse therapy with methylprednisone as well as trials with methotrexate and cyclophosphamide. Treatment with IVIG every 3 wk led to gradual clinical improvement over a 6-mo period, and an impressive fall in serum creatine kinase, and permitted conversion of her daily high-dose-prednisone regimen to a lower dose administered on alternate days.

\section{SUMMARY}

The studies cited herein highlight the potential benefits of IVIG therapy in a group of neurological disorders that are associated with 
aberrant immune responses. Indeed, all of the disorders discussed, except epilepsy, are associated with autoreactivity. The trials are preliminary and short-term and, except for idiopathic CIDP, uncontrolled. Interpretation of the findings of these uncontrolled studies is complicated by the fact that the natural history of all of these disorders is to show fluctuations. IVIG appears to be a potentially useful and safe agent in the treatment of patients with $M G$, intractable epilepsy, MS, and CIDP. Its place in the therapeutic approach to these neurological diseases must await the completion of controlled trials. Since other therapeutic modalities have already proven to be useful in several of these disorders, it will be important to determine if IVIG is more efficacious, safer, and more cost-effective. It is also worth considering whether the combination of IVIG and any of these more traditional approaches would provide added therapeutic benefit.

\section{REFERENCES}

1. Bussel, J. B. (1989), J. Allergy Clin. Immunol. 84, 566-578.

2. Pollock, S., Cunningham-Rundles, C., Smithwick, E. M., Barandun, S., and Good, R. (1982), N. Engl. J. Med. 307, 253.

3. Sultan, Y., Kazatchkine, M. D., Maisoneuve, P., and Nydegger, U. E. (1984), Lancet 2, 765-768.

4. Newburger, J. W., Takahashi, M., Burns, J. C., Beiser, A. S., Chung, K. J., Duffy, C. E., Glode, M.P., Mason, W. H., Reddy, V., Sander's, S. P., Shulman, S. T., Wiggins, J. W., Hicks, R. V., Fulton, D. R., Lewis, A. B., Leung, D. Y. M., Colton, T., Rasen, F. S., and Melish, M. E. (1986), N. Engl. J. Med. 315, $341-347$.

5. Levinson, A. I., Zweiman, B., and Lisak, R. P. (1987), e. Clin. Immunol. 7, 187-197.

6. Gadjos, P., Outin, H. D., Elkharray, D., Brunel, P., DeRohan-Chabot, P., Raphael, J. C., Goulon, M., Goulon-Gorau, C., and Morel, E. (1984), Lancet $1,406,407$.

7. Gadjos, P., Outin, H. D., Morel, E., Raphael, J. C., and Goulin, M. (1987), Ann. NY Acad. Sci. 505, 842-844.

8. Fateh-Moghadam, A., Wick, M., Besinger, U., and Guresen, R. G. (1984), Lancet 1, 848,849.

9. Besinger, U. A., Gateh-Moghadam, A., Knorr, H., Wick, M., Kissel, H., and Albiez, M. (1987), Ann. NY Acad. Sci. 505, 828-831.

10. Arsura, E. L., Bick, A., Brunner, N. G., Namba, T., and Grob, D. (1986), Arch. Intern. Med. 146, 1365-1368.

11. Cook, L., Howard, J. F., and Folds, J. D. (1988), J. Clin. Immunol. 8, 23-31. 
12. Basta, M., Ianglios, P. F., Margues, M., Frank, M. M., and Fries, L. F. (1990), Blood 74, 326-333.

13. Kawada, K. and Terasaki, P. I. (1987), Exp. Hematol. 15, 133-136.

14. Stohl, W. (1986), eJ. Immunol. 126, 4407.

15. Klinman, D. M. and Steinberg, A. D. (1986), Arthritis Rheum. 29, 697-705.

16. Hahn, B. H. and Ebling, R. M. (1984), J. Immunol. 132, 187-190.

17. Dietrich, G. and Kazatchkine, M. D. (1990), J. Clin. Invest. 85, 620-625.

18. Rossi, F. and Kazatchkine, M. D. (1989), J. Immunol. 143, 4104-4109.

19. Leung, D. Y. M. (1989), J. Allergy Clin. Immunol. 84, 588-595.

20. Karpak, S. E., Graf, L., and Rapport, M. M. (1976), Science 194, 735-737.

21. Benson, M., Blennow, G., and Rosen, I. (1987), Acta Paediatr. Scand. 76, $147-150$.

22. Seager, J., Jamiuson, D. L., Wilson, J., Hayward, A. R., and Soothill J. F. (1975), Lancet 2, 632-635.

23. Duse, M., Tibert, S., Plebani, M., Avanzini, M. A., Gardenghi, M., Menegati, E., Monufgo, V., and Ugazio, A. G. (1986), Monogr. Allergy 20, 128-134.

24. Plebani, A., Avanzini, M. A., Gardenghi, M., Menegati, E., Monufo, V., and Ugazio, A. G. (1988), Monogr. Allergy 23, 204-215.

25. Pechadre, J. C., Sauvesie, B., Osier, C., and Gilbert, J. (1977), Rev. EEG Neurophysiol. Clin. 7, 443-447.

26. Laffont, F., Esnasult, S., Gilbert, A., Peytour, M. A., Cathola, H. P., and Eygonnet, J. P. (1979), Ann. Med. Interne (Paris) 130, 307-312.

27. Ariizume, M., Shihara, H., Hibio, S., Ryo, S., Babak, Ogawa, K., Suzuki, Y., and Momoki, T. (1983), Lancet 2, 162,163.

28. Ariizume, M., Baba, K., Ogawa, K., et al. (1984), Hihon Univ. J. Med. 26, 229-242.

29. Echenne, B., Parayre-Chanez, M. J., Clat, J., and Baldy Moulkinier, M. (1987), Neuropediatrics 18, 126.

30. Sandstedt, P., Kostulas, V., and Larsson, L. E. (1984), Lancet 2, 1154,1155.

31. Schwartz, S. A. (1989), J. Allergy. Clin Immunol. 84, 603-607.

32. Hartung, H. P., Heininger, K., Schafer, W. et al. (1988), Advances in Neuroimmunology (Raine, C., ed.), Ann. NY Acad. Sci. 540, 122-161.

33. Dyck, P. J., Daube, J., O'Brien, P. C., et al. (1986), N. Engl. J. Med. 314, $461-465$.

34. Kleyweg, R. P., van der Meche, F. G. A., and Meulstee, J. Neurology 38, 1639-1641.

35. Vermeulen, M., van der Meche, F. G. A., Speelman, J. D., Weber, A., and Busch, H. F. M. (1985), J. Neurol. Sci. 70, 317-326.

36. Faed, J. M., Day, M. B. B. S., Pollock, M., Taylor, P. K., Nukada, H., and Hammond-Tooke G. D. (1989), Neurology 39, 422-425.

37. Albala, M., McNamara, M. E., Sokol, M., and Wyshock, E. (1987), Arch. Neurol. 44, 248,249.

38. van Doorn, P. A., Brand, A., Strengers, P. F. W., Meulstee, J., and Vermeulen, M. (1990), Neurology 40, 209-212.

39. Dalakas, M. C. and Engel, W. K. (1981), Ann. Neurol. 9(Suppl.), 134-145. 
40. Cook, D., Dalakas, M., Galdi, A., Biondi, D., and Porter, H. (1990), Neuro$\log y$ 40, 212-214.

41. van Doom, P. A., Brand, A, and Vermeulen, M. (1988), Neurology, 38, 15921595.

42. McFarland, H. F. (1988), Advances in Neuroimmunology (Raine, C., ed.), Ann. NY Acad. Sci. 540, 99-105.

43. Rothfelder, U., Neu, I., and Pelka, R. (1982), Wochenschr. Med. 124, 7478.

44. Schuller, E. and Govaerts, A. (1983), Wer. Neurol. 22, 205-212.

45. Soukop, W. and Tschabitscher, H. (1986), Wien Med. Wochenschr. 136,477480.

46. Henrikson, K. G. and Lindvall, B. (1990), Prog. Neurobiol. 35, 181-193.

47. Roifman, C. M., Schaffer, F. M., Wachsmuth, S. E., Murphy, G., and Gelfand, E. W. (1987), JAMA 255, 513-515. 